\title{
Bandgap calculation of a 2-D photonic crystal and its application towards a thin film solar cell
}

\author{
Md. AhsanHabib, Mohammad ZahirUddinSuja*, SunaynaBinte Bashar and Saeed Mahmud Ullah \\ Department of Applied Physics, Electronics \& Communication Engineering, \\ University of Dhaka, Dhaka-1000, Bangladesh
}

Received October 17, 2011; accepted November 20, 2011; published December 31, 2011

\begin{abstract}
Two-dimensional (2-D) photonic crystals (PhCs) of a square lattice have been investigated to find the complete photonic band gap. Band diagram calculations have been performed using the simulator "PWE band solver" of the software package optiFDTD ${ }^{\mathrm{TM}}$, based on a plane wave expansion method. Air holes in the dielectric background with four different shapes have been considered. Parameters like filling fraction, rod/air hole orientation angle and material dielectric constant have been varied. It has been found that $\mathrm{PhCs}$ with square air holes have the largest gap; the gap-midgap ratio is $13.66 \%$ for GaAs (when filling fraction, $f=0.680$ and orientation angle, $\theta=30^{\circ}$ ). Finally, a proposal is made to improve the efficiency of a thin film solar cell using this photonic crystal as a back reflector.
\end{abstract}

A photonic crystal is composed of a periodic arrangement of dielectric material in one, two or three dimensions. If the periodicity and symmetry of the crystal and the dielectric constants of the materials used are wellchosen, the band structure of such a crystal shows the photonic band gap (PBG) for one or both polarizations, i.e. at particular frequencies light propagation is prohibited in any direction in the crystal [1]. If, for some frequency range, a photonic crystal prohibits the propagation of electromagnetic waves of any polarization traveling in any direction from any source, we say that the crystal has a complete photonic band gap [1]. A triangular lattice has a large complete photonic band gap but it is difficult to fabricate [1]. So our attention goes to a square and a rectangular lattice. A 2-D periodic rectangular array of elliptic or circular air holes or dielectric rods in a background material can open a large complete PBG [2][3] but given the lattice symmetry, the largest absolute photonic band gap is achieved by selecting a scatterer of the same symmetry; e.g.; hexagonal rods in triangular or honeycomb lattices, square rods in square lattices, and rectangular rods in rectangular lattices [4].

In a thin film solar cell, photonic crystal applied as a back reflector has introduced a significant improvement in efficiency. It is working as the best solution for the advanced light trapping scheme, enhancing the optical path length by several orders of magnitudes [5]-[6]. Recent studies have shown that a thin film solar cell with a textured photonic crystal back reflector has greater efficiency than the one with a conventional metallic back reflector [5]. Further study has been carried out using a 2-

*E-mail: zahir.suja@yahoo.com
D photonic crystal with a triangular lattice of air holes made of photovoltaic material instead of a textured photonic crystal [6].

In this paper, a square lattice with various types of scatterer in a dielectric background has been considered and the effect of various parameters on a complete band gap has been investigated. In our calculation, the dielectric medium, GaAs with the relative dielectric constant $\varepsilon=11.4$ [7] has been used and simulated for the band gap, then other dielectric materials (e.g. Si, InP, Ge etc.) have been investigated in place of GaAs, keeping the parameters like filling fraction, $f$ and air hole orientation angle, $\theta$ unchanged (Fig 1 shows the definition of angle $\theta$ ). "PWE band solver" from the software package of optiFDTD $^{\mathrm{TM}}$ has been used for the band diagram calculation. "PWE band solver" is a band diagram calculator based on a plane wave expansion method.

Finally, focus is drawn towards the photonic crystal structure of the reflector. The crystal structure of a square lattice with square air holes in the $\mathrm{Si}$ background is proposed instead of a triangular lattice with circular air holes. In this structure a larger complete band gap is found. This structure can be used as a back reflector in place of a triangular lattice of circular air holes, which has no complete band gap [6] and also possesses fabrication difficulties [1].
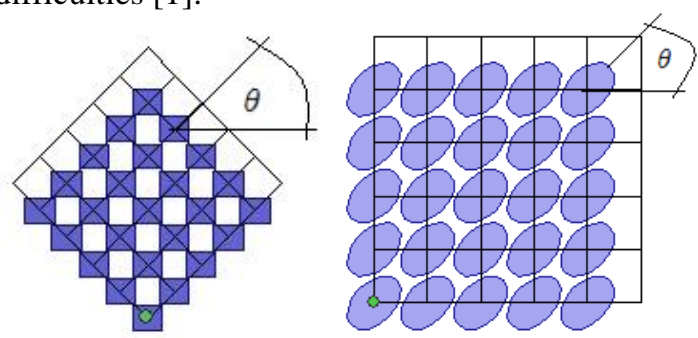

Fig. 1. Definition of dielectric rod/air hole orientation angle, $\theta$. (a) Rectangular dielectric rod/air hole. (b) Elliptical dielectric rod / air hole.

A square lattice with air holes of four different types has been considered, including square, rectangular, circular and elliptical air holes in the dielectric background. The results for different types of scatterer are listed as follows: 
- Square air holes in the dielectric background

For this structure it has been found that when the filling fraction, $f=0.68$ and the air hole orientation angle, $\theta=30^{\circ}$, the structure exhibits the maximum band gap width $(\Delta \omega)_{\max }=0.0600(2 \pi c / a)$ and normalized width of $\Delta \omega / \omega_{g}=13.66 \%$ for GaAs [see Fig. 2(a)], and $(\Delta \omega)_{\max }=0.055(2 \pi c / a)$ for Si [see Fig. 2(b)]. Here $\omega_{g}$ is the mid gap frequency, $a$ is the lattice constant and $c$ is the velocity of light in vacuum. Photonic crystals have the maximum band gap when the symmetry of air holes/dielectric rods is the same as the Brillouin zone (BZ) of the lattice. For a square lattice the shape of the Brillouin zone (BZ) is square and square air holes have the same symmetry as those of the BZ. For this reason the $\mathrm{PhCs}$ with a square air hole have the largest bandgap.
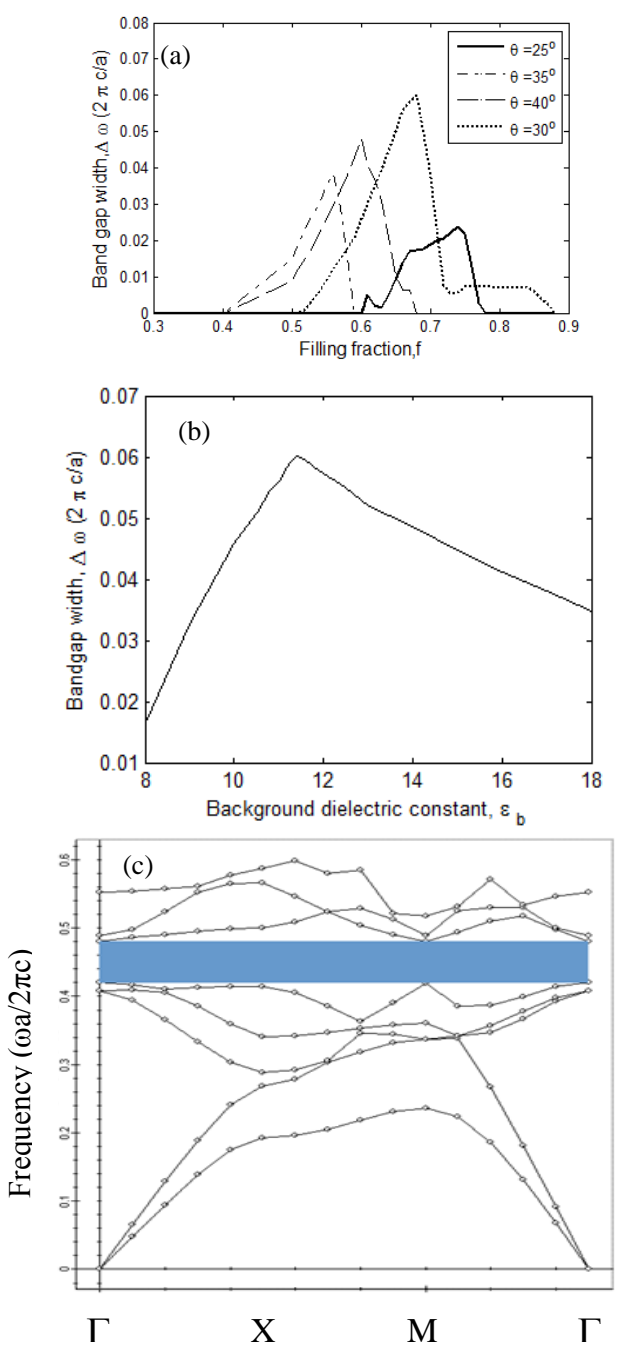

Fig. 2. Square air hole in the dielectric background. (a) Dependence of the band gap width, $\Delta \omega$ on the filling fraction, $f$ for four different rotation angle $\theta=25^{\circ}, 30^{\circ}, 35^{\circ}, 40^{\circ}$. (b) Dependence of bandgap width on dielectric constant keeping $f$ and $\theta$ constant $\left(f=0.68, \theta=30^{\circ}\right)$. (c) Band structure with a complete photonic band gap for the filling fraction, $f=0.68$, air hole orientation angle $\theta=30^{\circ}$ and hybrid polarization (TE and TM).
- Rectangular air holes in dielectric background

For this structure the maximum band gap width $(\Delta \omega)_{\max }=0.0336(2 \pi c / a)$ and normalized width of $\Delta \omega / \omega_{g}=4.17 \%$ for GaAs [see Fig. 3(a)], and $(\Delta \omega)_{\max }=0.0325(2 \pi c / a)$ for Si [see Fig. 3(b)] when filling fraction, $f=0.576$ and air hole orientation angle, $\theta=45^{\circ}$ and $L_{a} / L_{b}=0.84$ where, $L_{a}$ and $L_{b}$ denote length and width of the rectangle respectively. Although, rectangular scatters are less symmetrical than the square scatters but due to the difference in the shape from the square Brillouin zone of square lattice, rectangular air holes in dielectric background have the less band gap than the square one.
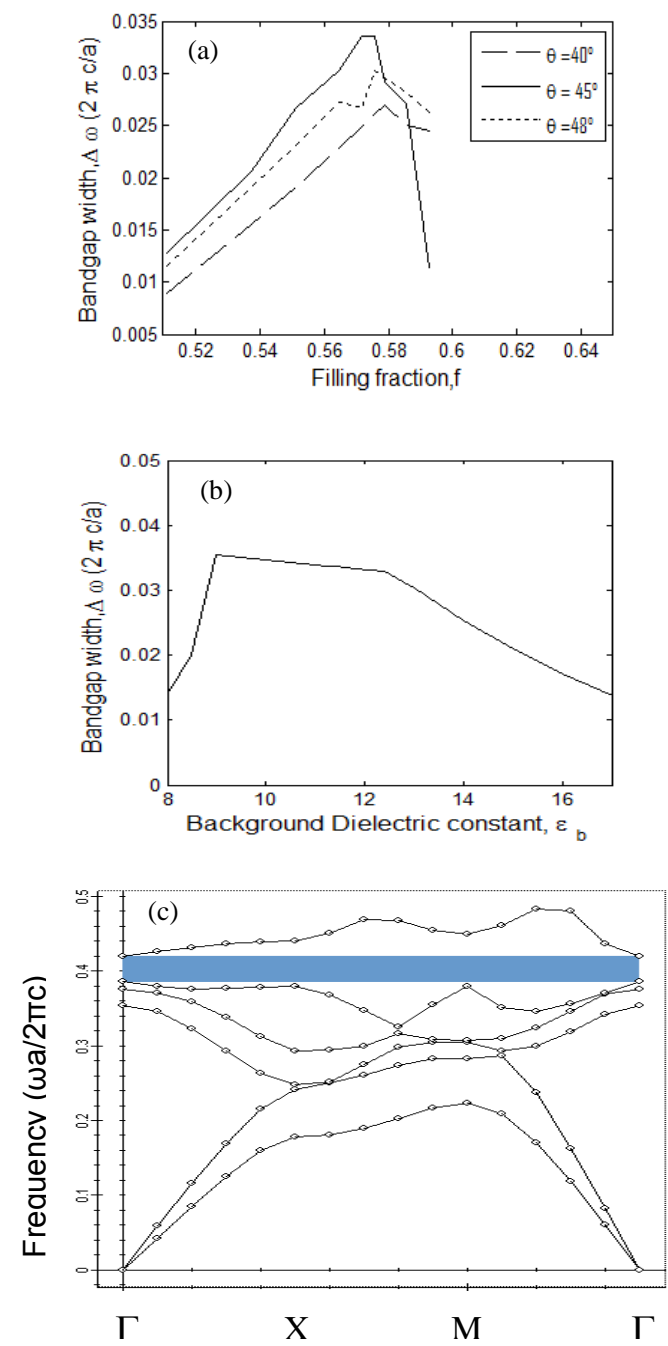

Fig. 3. Rectangular air hole in the dielectric background.(a) dependence of the band gap width, $\Delta \omega$ on the filling fraction, $f$ for three different rotation angle, $\theta=40^{\circ}, 45^{\circ}, 48^{\circ}$. (b) Dependence of bandgap width on dielectric constant keeping $f$ and $\theta$ constant $\left(f=0.576\right.$ and $\left.\theta=45^{\circ}\right)$. (c) Band structure with complete photonic band gap for the filling fraction $f=0.576$, air hole orientation angle $\theta=45^{\circ}$ and hybrid polarization. 
- Circular and elliptical air holes in the dielectric background

For a circular air hole, the maximum band gap width $(\Delta \omega)_{\max }=0.004(2 \pi c / a)$ and the normalized band gap width $\Delta \omega / \omega_{g}=0.78 \%$ at $f=0.76$ have been found. For elliptical air holes it has been found that the largest band gap width, $(\Delta \omega)_{\max }=0.0101(2 \pi c / a)$ and the normalized bandgap width $\Delta \omega / \omega_{g}=1.71 \%$ at $f$ $=0.7236$ and $\theta=20^{\circ}$. Figure 4 shows the band diagram for circular and elliptical air holes. For these cases a small band gap has been found because elliptical and circular scatters are more symmetrical than the square and rectangular air hole. Since these two structures show a small bandgap, the details are not shown here.

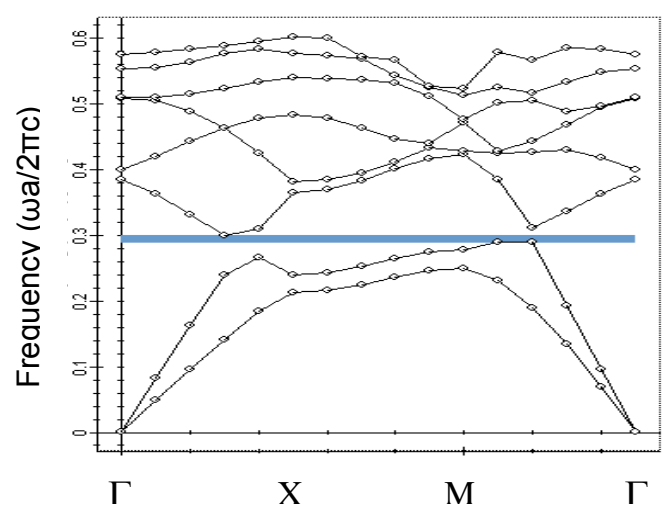

(a)

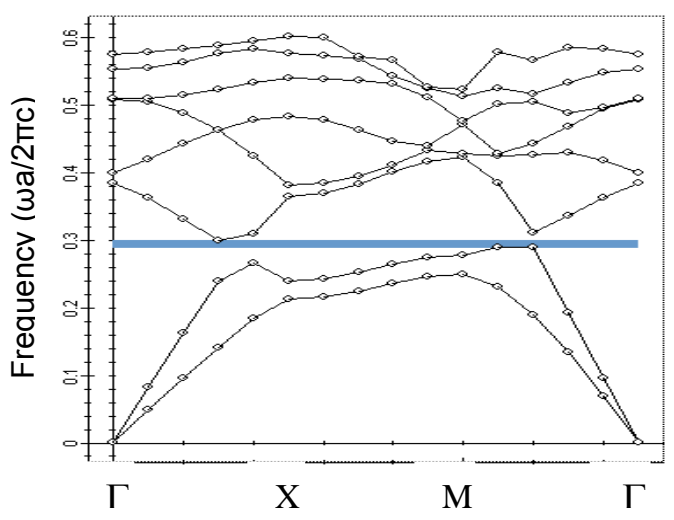

(b)

Fig. 4. Band structure with a complete photonic band gap. (a) Circular air hole in the dielectric background. (b) Elliptical air hole in the dielectric background with hybrid polarization.
In the present paper, $\mathrm{PBG}$ for four different geometric shapes of air holes in a square lattice have been investigated. Among them, the square air holes in the dielectric back ground have shown the largest complete photonic band gap. For the filling fraction $f=0.68$ and the air hole orientation angle $\theta=30^{\circ}$, the structure exhibits the maximum band gap width $(\Delta \omega)_{\max }=0.055(2 \pi c / a)$ for $\mathrm{Si}$, which is significantly larger than any other structures. Since, it is a complete band gap; it will work as a perfect reflector for both TE and TM mode of incident light that falls within this band gap. So, for the light waves having the frequencies within this band gap, an incident from air at any angle acts as a perfect reflector with the reflectivity about $100 \%$ for either or both polarizations [1], whereas no metallic reflector has the reflectivity of more than $80 \%$. Additionally, frequencies outside the photonic bandgap are refracted into modes with a high density of states [8].

A recent study has used triangular air holes in the dielectric background as a back reflector of the solar cell [6]. But this crystal structure exhibits a significant band gap only for TE mode but not for TM mode. Here the paper proposes a square lattice of square air holes in the dielectric background as a back reflector in place of a triangular lattice of air holes, which could work better as a reflector because it has a sufficiently large complete band gap. If it were possible to reflect TM mode along with TE mode back into the solar cell, we think, it would increase the light conversion efficiency of a thin film solar cell.

\section{References}

[1] J.D. Joannopoulos, S.G. Johnson, J. Winn, R. Meade, Photonic Crystals: Molding the Flow of Light (Princeton University Press, New Jersey 2008).

[2] L. Zeng, Y. Yi, C. Hong, J. Liu, N. Feng, X. Duan, L.C. Kimerlin, B.A Alamariu, Appl. Phys. Lett. 89, 111111 (2006).

[3] P. Bermel, C. Luo, L. Zeng, L.C. Kimerling, J.D. Joannopoulos, Opt. Exp. 1516986 (2007).

[4] M. Qiu, S. He, Phys. Rev. B 60, 10610 (1999).

[5] G. Qiua, F. Lina, Y.P. Licompelte, Complete two-dimensional bandgap of photonic crystals of a rectangular Bravais lattice (Elsevier Science, 2003).

[6] R. Wang, X.H. Wang, B.Y. Gu, G.Z. Yang, J. Appl. Phys. 90, 4307 (2001).

[7] J. Gee, 29th IEEE Photovolt. Spec. Conf., pp. 150-153 (2002).

[8] J.I. Pankove, Optical Process in Semiconductors (Dover, New York 1971). 